APPENDIX 1 : POSTER PAPERS 


\section{DISTORTION OF COSMIC MICROWAVE BACKGROUND RADIATION AND DECAY OF WEAKLY INTERACTING PARTICLES}

Katsuhiko Sato and Masahiro Kawasaki
Department of Physics, Faculty of Science,
The University of Tokyo, Tokyo 113 Japan

Recent1y Nagoya-Berkeley group (Matsumoto et al, 1987) observed microwave radiation in the Wien region precisely and found the excess of temperature. In the previous paper (kawasaki and Sato,1986), we investigated the distortion of the spectrum of microwave background radiation due to the radiative decay of weakly interacting massive particles. At that time, however, the distortion was not observed. In the present paper, we investigated in detail whether the decay of WIMPs can account for the observed distortion of the spectrum or not. In Fig. 1 , an example is shown.

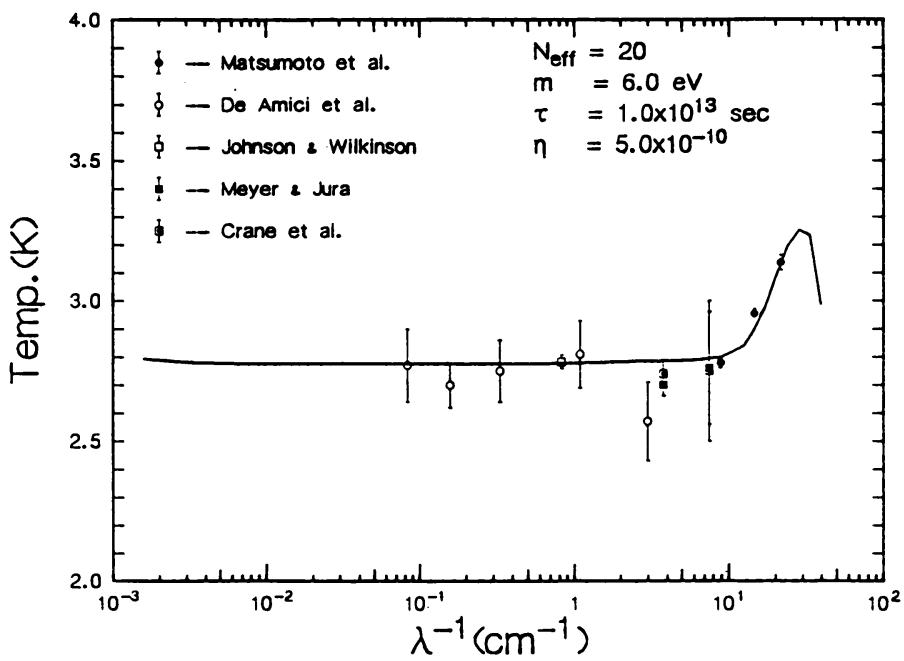

Fig. $1_{13}$ The spectrum distorted by the decay of WIMPs with the lifetime, $1.0 \times 10^{13} \mathrm{sec}$, mass, $6.0 \mathrm{eV}$ and $\mathrm{N}_{\text {eff }}=20$, where $\mathrm{N}_{\text {eff }}$ is the statistical weight of particles when the WIMPS decouple from the matter in the early universe.

We found that WIMPs, with appropriate lifetime, $10^{12}<\mathrm{t}<10^{14} \mathrm{sec}$, mass, $\mathrm{m} 2 \times 10^{7}(\mathrm{t} / 1 \mathrm{sec})^{-1 / 2} \mathrm{eV}$, and the number density, $1 / 10-1 / 20 \mathrm{x}$ (the photon number density) can account for the observed distortion. However, such strong coupling to photons is stringently constrainted from the stellar cooling due to the WIMPs. Details, see, Kawasaki and Sato(1987).

\section{References}

Kawasaki, M. and Sato, K. 1986, Phys. Lett. B169, 280.

Kawasaki, M. and Sato, K. 1987, preprint UTAP-59/87.

Matsumoto, T. et a1, 1987, submitted to Astrophys. J. Lett. 509 